\title{
CHRONIC MYCOTIC-ASSOCIATED SURGICAL NAIL PATHOLOGY COMPLICATED WITH INGROWN NAIL (NAIL INCARNATION): THE ANALYSES OF CLINICAL CASES AND COMPLEX TREATMENT
}

\author{
A. R. Vergun, B. M. Parashchuk, M. R. Krasnyy, Z. M. Kit, O. M. Vergun \\ DANYLO HALYTSKY LVIV NATIONAL MEDICAL UNIVERSITY, LVIV, UKRAINE
}

Background. Conservative treatment of secondary recurrent unguis incarnatus are not very effective and Dupuytren's method, Emmert-Schmiden surgeries etc. are very traumatic, disfigure nail bone, distort anatomic and functional unity of a nail.

Objective. The aim of our research was the optimal sequence of holiatry, surgical moving away of nails, local and system therapy after excision of the staggered nails in case of destructive onychomycosis complicated by secondary ingrown nail for some patients with complicated mycotic defeat of nails. Clinical options of surgical treatment were clarified; morphologic changes were studied; causes of unsatisfactory outcomes of chronic pathology complex treatment were analyzed for prospective approaches to preventing of relapses.

Methods. The analysis justifies the feasibility of establishing of predictive relationships between clinical variants of chronic purulent necrotic infections and combined comorbidity. System therapy by itraconazole before operative treatment (basic onychial defeats sanation) and in a postoperative period was carried out. Types of operative treatment applied may be divided into five statistic groups.

Results. Over a five-year period (2010-2015) 436 unguis incarnates diagnosis (325 cases of incarnated onychomycosis) in 259 men and 177 women aged 12-67 were performed, 38 of them with incarnated onychogryphosis had diabetes mellitus and 24 had metabolic syndrome. Analysis of subonychial scraping allowed stating the prevalence of red trichophytia in $31 \%$ of cases with the bacterial flora. Patients with combined pathology got 4-5 five-day system 'pulses' of 400 mg/day itraconazole therapy. In patients with primary advantage of providing low-impact methods of nail excision with access via onycholisation structure a nail extirpation and marginal matricectomy was carried out; dermatophytoms and stratification on a nail bed were deleted.

Conclusions. Type of onychectomy wound cytograms in the study group on the $10^{\text {th }}$ day was defined as regenerative-inflammatory in $24.81 \%$, regeneratory in $75.19 \%(p=0.031)$. The methods of surgical treatment of mycotical recurrent nail incarnation have been improved considering patho- and morphogenetic properties of destructive onychomycosis.

KEY WORDS: destructive onychomycosis; secondary recurrent ingrown nail; antimycotic therapy; surgical nail removal.

\section{Introduction}

Dermatophytes, infecting a nail matrix, were determined as the dystrophic changes of nail and a subnail hyperkeratinization and dermatophytoms [2, 3], surgical nail pathology, that compress a nail that is the driving nosotropic member of the secondary ingrowing and has an influence on curative tactics [4], in particular on the necessity of the surgical moving away $[8,21]$. The pathogenetic links leading to increase of the probability of occurrence and

Corresponding author: Andriy Vergun, Department of Outpatient Care, Family Medicine and Dermatology, Venerology, Danylo Halytsky Lviv National Medical University, 1 Konovaltsya Street, Lviv, Ukraine, 79013

Phone number: +380322757632

E-mail: plagiamail@meta.ua development of feet mycosis and onychomycosis for diabetic patients include the pathology of cardiovascular and nervous systems [10], disruption of glycolysis, resulting in lower energy supply of skin cells and changes in metabolism, skin dysfunction, determining rapid progression and chronic mycosis $[18,20]$.

The objective was to study certain peculiarities of mycosis-associated pathology and treatment, including surgical removal of nails in patients with onychogryphosis and recurrent ingrown with underlying diabetes mellitus [20]. The results of determining of vitamins $B_{1}, B_{2}$, PP, $\mathrm{B}_{6}$ and $\mathrm{C}$ in blood and their derivatives are analysed [22]. Some 50-year-old patients, who have high mycotic surgical nail pathology, have 
the endothelial dysfunctions and polyvitamin disturbances that are some factors from clinical corrections $[10,12,22]$.

The study involves peculiarities of treatment, some indicators of lipid exchange of patients with diabetes mellitus $[9,10,18,20]$, metabolic syndrome accompanied by destructive onychomycosis and secondary nail ingrowth. Clinical options of in-patient and out-patient surgical treatment for nail ingrowth (mono-lesions; complicated and combined mycotic-associated processes and relapses) were clarified; morphologic changes were studied; causes of unsatisfactory outcomes of chronic pathology complex treatment were analysed for prospective approaches to preventing relapses.

If the infection involves the eponychium as well as the lateral fold, it is called eponychia [15, 17]. Its extension to the opposite side of the fingernail, which is uncommon, is called runaround abscess. In these cases, the paronychia is compressed along the nail edge, trapping the abscess. All procedures that successfully treat paronychia separate it from the hard nail [23, 24]. If the infection is limited to less than onehalf of the eponychium, a single incision placed to drain the paronychium and to elevate the eponychial fold for excision of the proximal onethird of the nail is satisfactory. If the entire eponychium is involved, two incisions are required. The relevance of the problem of the ingrown nail (onychocryptosis, unguis incarnatus) in an urgent outpatient purulent surgery is caused by the increased frequency of its occurrence, chronicity, complications, not uncommon early and late postoperative relapses [8, $15,17]$. Conservative and orthopedic treatment of incarnatus surgical nail pathology are not very effective while Dupuytren's method, Emmert-Schmiden surgeries etc. are very traumatic, disfigure nail bone, $[4,5,21,24]$ distort anatomic and functional unity of a finger and in $2-20 \%$ of cases (depending on absence or presence of onychocryptosis and fungal agents) cause a relapse.

The clinical features and ways to optimize the treatment of patients with onychomycosis and destructive secondary ingrown nails are clarified in the article. The results of complex treatment of the patients with nail trichophytosis, associated with ingrown toenail, submitted according to dependence on a nail plate and eponycheal changes are presented in the publication.

The aim of our research is the optimal sequence of holiatry, surgical moving away of nails, local and system therapy after moving away the staggered nails in cases of destructive onychomycosis complicated by the secondary ingrown nail for some patients with the complicated mycotic defeat of nails.

\section{Methods}

Certain peculiar features of the clinical course and comprehensive treatment were studied, including surgical removal of the nails in patients with onychogryphosis associated with an ingrown nail (onychocryptosis, nail incarnation). Results of ingrown nail surgical correction were studied to improve the results of complex treatment. Over a five-year period (2010-2015) 436 unguis incarnates diagnosis (325 cases of incarnated onychomycosis) in 259 men and 177 women aged 12-67 were performed. In 182 patients late relapses of onychocryptosis were confirmed after previous surgeries at other clinics. Conservative treatment was recommended only at early stages of ingrowth. Removal of the affected nails was performed for patients with mycotic lesions (local and systemic fungicide therapies were used). Investigation of the morphogenesis of destructive aspect of the mycotic lesions was carried out. A variety of factors, aetiology, and pathogenesis of chronic purulent necrotic lesions of the foot, contributing to the occurrence, progression and recurrent course of these diseases, creates objective difficulties of diagnosis $[14,16,19]$. The analysis justifies the feasibility of establishing predictive relationships between clinical variants of chronic purulent necrotic infections and combined comorbidity $[1,25]$. System therapy by itraconazole in operative treatment (basic onychial defeats sanation) and in a postoperative period was carried out $[12,25]$. The applied types of operative treatment of surgical nail pathology may be divided into five main groups: 1 - EmmertSchmiden type surgeries (marginal excision of nail plate and eponychia with marginal removal of the growing part via partial matricectomy); 2 - Dupuytren's type surgeries (onychectomy - complete removal of nail plate); 3 Bartlett type surgeries (local tissue plastic reconstruction); 4 - marginal resection of marginal section of nail plate; 5 - Meleshevych surgery; 6 - our modifications (with previous block-type eponychectomy).

\section{Results}

Over the five-year period we examined and treated 98 patients of 52-86 years old with in- 
carnated onychogryphosis (Fig. 1): 67 men $(68.37 \%)$ and 31 women $(31.63 \%)$ of the examined patients, 38 of them had diabetes mellitus and 24 had metabolic syndrome. Three variants of dermatophytoma are differentiated: front centre, with up to $25 \%$ of eroded nail - 45 cases, subtotal, from 25 to $70 \%$ (without capturing the growth plate) - 38 cases, total from 70 to $90 \%$ (with affected growth plate of the nail) - the other 15 cases. In all cases, dermatophytoma affected distal and central part of the nail bed. Analysis of subonychial scraping allowed stating the prevalence of red trichophytia $[3,4]$ : in $74 \%$ of cases it was associated with mold, in $26 \%$ of cases - with yeast fungi; in $31 \%$ of cases with bacterial flora [1].

Conglomerate of nail plate and subungual hyperkeratosis and trichophytosis calcinated completely, forming onychogryphosis with deformation [6, 7] (Fig. 2) and secondary recurrent ingrown nail $[15,16,22,23]$.

In patients with onychomycosis, especially in severe destructive forms of subungual hyperkeratosis, large deterioration of microcirculation was noted. Rheographic prevailed spas-

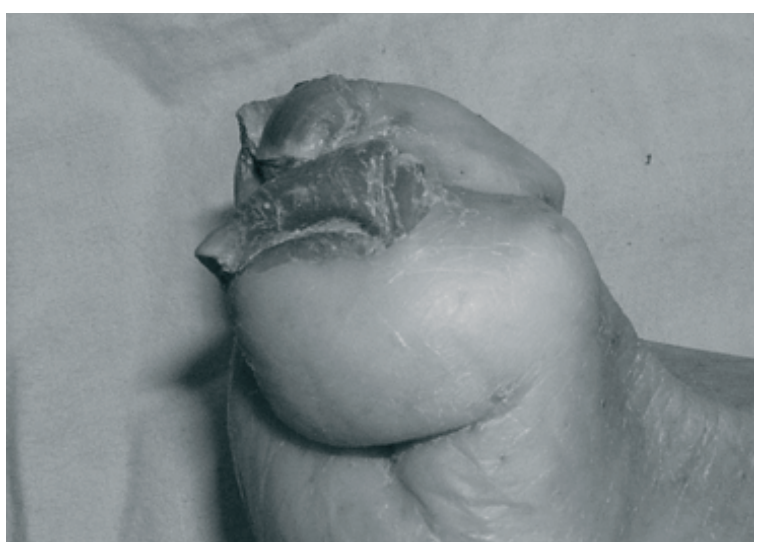

Fig. 1. Trichophytial polyonychomycosis, onychogryphosis. Clinical case. 81-year-old woman.



Fig. 2. Total nail dermatophytosis (Tr. Rubrum), polyonychomycosis. Big polyonychial gryphosis. Clinical case. 82-year-old man. tic type curves $(p<0.01)[9,10]$. Index of open capillaries was reduced by $31 \%$. Patients in both groups mainly were affected with hallucis on left foot - in 188 people, the other - on right foot (133 patients), the presence of pathological ingrown nail plates of hallucis of both feet [12, $15,17]$ and otherfingers were examined in other patients.

Mycotic associated hyponychial panaritium was diagnosed in 16 patients with onychogryphosis (16.33\%), purulent paronychia was diagnosed in 11 patients (11.22\%), the other 5 patients of this group suffered from eponychial abscess (5.10\%). Patients of the main group underwent a three-day adjuvant systemic fungicide therapy: $400 \mathrm{mg}$ itraconazole daily, during 4 days (the first 2 postoperative days) as pulse therapy. A similar dose at weekly intervals was carried out following five-day 2-3 cycle pulse $[23,25]$.

Removal of the affected nails for patients with polyonychomycosis was performed in successive stages at add-back of certain systemic 'pulses' with itraconazole. Patients with such combined pathology got 4-5 five-day system 'pulses' of $400 \mathrm{mg} /$ day itraconazole therapy together with a simultaneous use of hepatoprotectors and correction of comorbid pathology [21-23]. Provided adequate surgical treatment, in addition to standard decompression stage (complete removal of the nail plate), contained antirecurrent component to prevent from repeated ingrowth. Along with antimycotic therapy and correction of comorbid pathology the following procedures were carried out: cutting pathologic eponychial tissues, hypergranulations and necroses [22, 24] (Fig. 3); removing nail plate with partial marginal ma-

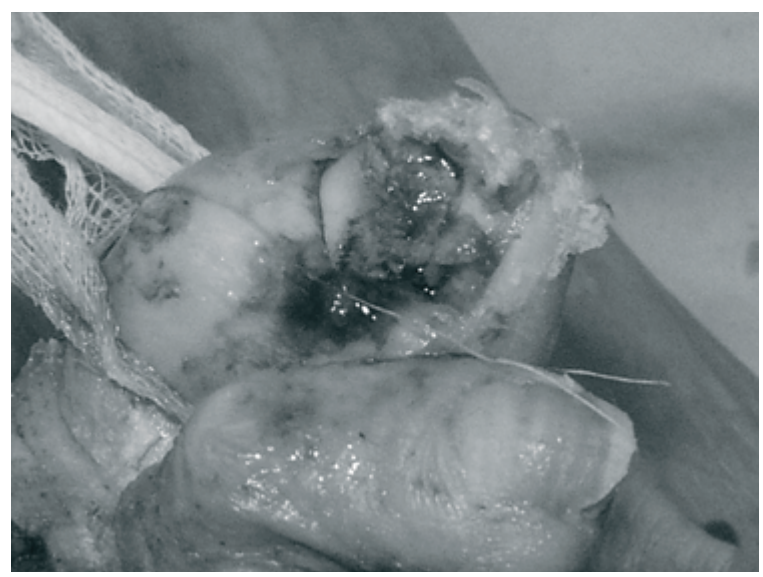

Fig. 3. Surgical nail pathology, subnail subungual hyperkeratosis and dermatophytosis, big dermatophytoma, hypergranulations and local necroses. Intraoperation photo. Trichophytial polyonychomycosis, incarnated onychogryphosis. Clinical case. 81-year-old woman. 
trixectomy in the ingrowth area $[2,3,20]$. Noninvasive methods of nail excision and marginal nail resection were preferred for patients with diabetes mellitus.

During the five-year period we examined and treated 38 patients with onychogryphosis and diabetes mellitus type 2 . We provide the research on the characteristic features of the pathological process to create the optimal scheme of complex treatment for patients with abnormal ingrowth of nail plate with underlying diabetes mellitus type $2[9,18]$.

23 patients with ingrown onychogryphosis and underlying diabetes mellitus, diabetic micro and macroangiopathy (prospective material, the treatment group), and onychogryphosis and recurrent incarnation of toenail (pathology being clinically dominant and manifesting through pain syndrome) and multiple destructive mycotic lesions of other nail plates of both feet: 14 men and 9 women, 55-80 years old were involved in the surgery. The other 15 people with onychogryphosis of the first toe (hallux) and fungus of other nails constituted the control group. The duration of clinicallymanifesting nail mycotic process in all studied cases exceeded 5 years. Mycosis-associated acute purulent pathology $[19,21,23]$ was confirmed in 13 patients ( $34.21 \%$ of the examined, $52 \%$ cases) of the treatment group and in 5 patients of the control group $(13.16 \%$ of the examined, $30 \%$ cases) correspondingly. Systemic adjuvant pulse therapy with $400 \mathrm{mg}$ itraconazole was applied during two days before the initial surgical treatment and during the first three days of postoperative period [19]. Remediation of other affected nails in order to prevent from mycosis reinfection was carried out with antimycotic amorolfine $5 \%$ lacquer and ciclopirox $8 \%$ nail lacquer solution $[2,11-13,22]$. Removal of other nails affected by hyperkeratosis with trichophytosis was performed through onycholysis by separate successive stages, where certain 'pulses' of therapy were supported with itraconazole [19, 21].

We prospectively examined 93 patients with metabolic syndrome $[9,10]$, associated with surgical nail pathology, destructive onychomycosis and secondary ingrown toenails. All patients underwent a comprehensive treatment of comorbid pathology, corrected using the systemic antimycotic therapy and terbinafine antifungal liniments, ciclopirox nail lacquer [11, 13] and surgical methods for removing nail plates $[8,9,12,22]$. We studied lipid metabolism that manifested biochemical change indicators in the lipid profile, as well as their relations, which were significantly higher in both groups of patients (the main and control group), $p<0.01$ for both groups; we evidenced the positive correlation between the level of total cholesterol and leptin $(p<0.01)$. The concentration of highdensity lipoprotein cholesterol in patients of the main group was $1.12 \pm 0.06 \mathrm{mmol} / \mathrm{L} \mathrm{com}$ pared with patients in the control group $1.07 \pm 0.06 \mathrm{mmol} / \mathrm{L}$. The average content of nitrogen oxide in the study group (metabolic syndrome) was higher than in the healthy patients $-15.06 \pm 0.97 \mathrm{mcmol} / \mathrm{L}, \mathrm{p}<0.05$.

Significant decrease of HOMA-index of $\beta$-cell function and increase of HOMA-index of insulin resistance $[18,20](10.21 \pm 1.9$ in the treatment group and $4.12 \pm 1.12$ in the control group, $p<0.01$ ) was detected in patients with underlying diabetes mellitus type 2 (the treatment group) with polyonychomycosis and trichophytosis onychogryphosis, and recurrent incarnation of the nail edge. High total cholesterol over $5.18 \mathrm{mmol} / \mathrm{l}$ was detected for all patients of the main group, i.e., $7.28 \pm 0.07 \mathrm{mmol} / \mathrm{l}$, and $5.45 \pm 0.12 \mathrm{mmol} / \mathrm{l}$ in the half of the control group; the deviation of laboratory parameters of low and high density lipoprotein cholesterol was also confirmed.

Polyonychomycosis and big trichophytic subungual hyperkeratosis with secondary nail ingrowth [6-9] were diagnosed in the main group comprising 62 patients with type 2 diabetes mellitus ( 48 males and 14 females, aged 42-65). Metabolic syndrome was confirmed in the comparison group comprising 53 patients with arterial hypertension and complicated mycotic nail damage. The rest 276 patients constituted the control group. We studied biochemical findings, lipid blood spectrum: total cholesterol, high-density lipoprotein cholesterol, low-density lipoprotein cholesterol; nitric oxide of blood plasma; leptin [9]. Lipid blood spectrum and nitric oxide level [10] were determined before and after pharmacotherapy along with continuous antihypertensive therapy (correction of intercurrent and comorbid pathology). The objective criterion of insulin resistance was the HOMA-IR index (the Homeostasis Model Assessment) involving glucose and insulin levels on an empty stomach divided by 22.5 coefficient. Patients of the main and the comparison groups with polyonychomycosis and trichophytic subungual hyperkeratosis with secondary ingrown nail experienced considerable decrease of HOMA-index of $\beta$-cells function and increasing HOMA-index of insulin resist- 
ance $(8.11 \pm 1.1$ in the main group, $5.89 \pm 2.1$ in the comparison group and $2.23 \pm 1.18$ in the control group, $\mathrm{p}^{1}=0,01 ; \mathrm{p}^{2}=0.01$ ). Considerable positive correlation between all indicators of carbohydrate exchange was identified in groups comprising patients with type 2 diabetes mellitus: glucose and insulin $(r=0.51$; $p=0.01)$, and the HOMA-index $(r=0.70 ; p=0.01)$, and glycolyzed haemoglobin $(r=0.75 ; p=0.001)$; insulin and the HOMA-index $(r=0.73 ; p=0.01)$ and glycolyzed haemoglobin $(r=0.65 ; p=0.01)$; the HOMA-index and glycolyzed haemoglobin $(r=0.67 ; p=0.01)$. Considerable increase of circulating insulin was identified directly after surgical treatment (in the main group and the comparison group correspondingly $15.33 \pm 0.23$ and $8.24 \pm 1.18 \mathrm{mkMO} / \mathrm{l}, \mathrm{p}=0.01$ ), the HOMA-index of insulin resistance $(p=0.05)$ and the HOMA-index of $\beta$-cells function $(p=0.05)$ in comparison with the control group with further tendency to some decrease in the process of treatment. We determined disturbances in lipid exchange, insulin resistance, lipid blood spectrum changes that were considerably higher in both groups of patients (the main and comparison groups), $p=0.01$ for both groups in comparison with the control group.

\section{Discussion}

On one hand the subungual hyperkeratosis and dermatophytosis caused compression of the central part of the nail, epionychium edges 'ingrew' to periungual walls thus recurrent ingrown nail was formed; on the other hand, constant compression caused destruction of central part of nail bed; this process was typical for $32(84.32 \%)$ cases. Methods of surgical treatment of uncomplicated onychogryphosis $[6,7]$ and onychogryphosis complicated with recurrent nail incarnation $[8,15]$ were improved considering patho- and morphogenetic properties of destructive onychomycosis $[16,17]$; removal of the mycotic affected nails of these patients should reasonably be conducted through onycholized structures with simultaneous removal of dermatophytoma, hyperkeratosis, and ingrowth areas with hypergranulation. Surgical treatment was performed according to the standard algorithm due to patholo gical eponychial changes in the patients with primary advantage of providing low-impact methods of nail excision with access via onycholisation structure and wedge resection of the nail $[2,20]$. When combined incarnation of onychomycosis and acute eponychial abscess comply with disclosure abscess, excision of abnormal tissue eponychial hypergranulation and focal necrosis, and removal of the nail plate, enlarged partial marginal matrixectomy in the ingrowth area are performed. When combined with onychomycosis and nail incarnation acute eponychial abscess, an autopsy ulcer, excision of abnormal tissue eponychial hypergranulation and focal necrosis, and removal of the nail plate, enlarged partial marginal matrixectomy in the ingrowth area are made. Other patients (the control group) underwent a typical nail removal - operations such as Dupuytren's: complete removal of the nail plate under the guise of 'classical' pulse therapy by itraconazole and terbinafine $[13,21]$; in 45 cases this intervention combined with simultaneous excision of the modified cuticle and plastic - operations such as Bartlett (plastic local tissue) and Meleshevich.

The processes of destruction of the nail in patients with trichophyton onychomycosis and type 2 diabetes mellitus / metabolic syndrome is much faster and is characterized by a more pronounced morphological variants of mycotic nail destruction that determine the occurrence of secondary ingrowth and attachment with intercurrent flora with the emergence of dermatophythoma with centres of decay and necrotic foci in the nail bed.

A nail extirpation and partial marginal matricectomy mechanical carving and diathermocoagulation with the further scraping off by the Folkman's spoon were carried out; dermatophytoms and stratification on a nail bed were deleted. Sanation of other nails for prevention of mycotic reinfection was carried out by ciclopirox nail lacquer [11]. Application of system enzymic protheolitical therapy allows to considerably improve the primary results of holiatry of the complicated subnail hyperkeratinization, diminish a perifocal edema and inflammation; stimulate the necrolitical and reparative processes in an operating wound that clinically shows up to the accelerations of its granulation and epithelization; accelerate cicatrization of surface, abbreviate the terms of temporal non-operability. For patients with type 2 diabetes mellitus (the main and comparison groups) with polyonychomycosis and dermatophytoms as well as secondary ingrown nail, subnail hyperkeratinization were performed for certain significant [20] reduction of the HOMAindex of function and the increase of the HOMAindex of insuline resistance. The chart of holiatry applied proved the effectiveness of treatment of bad resistance cases of destructive 
polyonychomycosis by a subnail hyperkeratinization and secondary ingrown nail, in particular for patients with the type 2 diabetes mellitus [9]

Arguing that the removal of the nail plate in the patients with destructive onychomycosis with secondary incarnation nail advantageously carried out through onycholisation structure with simultaneous correction of pathological bed changes and cuticle, which in combination with antifungal treatment provides positive dynamics of regenerative type cytologic picture and shorter healing onychectomy wounds in 18 - 27 days to $12-25$ days, with good early and long-term results. The type of transaction cytograms of onychectomy wounds in the study group on the $10^{\text {th }}$ day of post-operative period is defined as the regenerative-inflammatory in $24.81 \%$, as regenerative in $75.19 \%(p=0.031)$. In these embodiments, in the control group the indicators were respectively $53.12 \%$ and $46.88 \%$. The advantage of regenerative option of cytological picture proves the correct choice of treatment strategy and accelerates wound healing after removing nail in patients of the main group. The factors that influence the occurrence and progression of incarnations, and disease recurrence after surgical treatment require further study $[3,4]$.

We have studied the results of onychocryptosis surgeries of surgical type 1 correction in 84 patients, type 2 - in 66 patients, type 3 - in the other 50 cases, type 4 - in 42 persons, type 5 - in another 27 patients, type 6 - in 56 patients (with the use of our modification of surgical treatment). Three types of trichophytosis have been differentiated: frontal central - with erosion of up to $25 \%$ of nail area, subtotal - from 25 to $70 \%$ (without touching upon growth area), total - from 70 to $90 \%$ (with affected growth area). In 65 mycotic trichophitis patients with secondary nail incarnation a standard itraconazole pulse therapy was applied. The presence of onycholytic focuses and degradation of hyperkeratotic areas which result in lamination of a part of nail plate proves the feasibility of performing low-trauma onychectomy for patients with trichophyton onychomycosis with secondary incarnation via onycholized structure with the single-stage sequential removal of dermatophyte and ingrowth areas with changed eponychial folds. Relapse causes after Meleshevych, Emmert-Schmiden, Bartlett surgeries were technical faults of surgical tools, intraoperative nail bed trauma, faults of postoperative anti-relapse treatments, surgical area trauma, wearing tight shoes, non-compliance with doctor's recommendations for correction of orthopaedic pathology, onychomycosis.

We believe that the less traumatic removal of nails through onycholysis should be prefered, particularly after such treatment, the patients with diabetes mellitus experienced healing time of operative wound (crust formation) during 16-23 days (average healing duration is 19 days) and had the indices tend to the control group; indices of the patients with diabetes and 'classical' nail removal (onicectomia) were normal in 24-30 days (average healing duration is 26 days), indices of the control group - in 14-22 days (average healing duration is 18 days).

\section{Conclusions}

In all cases of mycotic onychocryptosis (secondary ingrown toenail) the patients underwent a comprehensive treatment of comorbid pathology corrected by the systemic antimycotic therapy and terbinafine antifungal liniments, ciclopirox nail lacquer and surgical methods for removing nail plates, supplemented the eponychial resections and partial marginal matrixectomy.

System therapy of itraconazole before operative treatment (basic onychial defeats sanation) and in a postoperative period was carried out. Patients with combined pathology got 4-5 five-day system 'pulses' of $400 \mathrm{mg} /$ day itraconazole therapy.

In patients with primary advantage of providing low-impact methods of nail excision with access via onycholisation structure a nail extirpation and partial marginal matricectomy mechanical carving and diathermocoagulation with the further scraping off by the Folkman's spoon were carried out; dermatophytoms and stratification on a nail bed were deleted. Sanation of other nails for prevention of mycotic reinfection was carried out by ciclopirox nail lacquer.

Methods of surgical treatment of uncomplicated onychogryphosis and onychogryphosis complicated with recurrent nail incarnation have been improved considering patho- and morphogenetic properties of destructive onychomycosis; removal of the mycotic affected nails of these patients should reasonably be conducted through onycholized structures with simultaneous removal of dermatophytoma, hyperkeratosis, and ingrowth areas with hypergranulation. Type of onychectomy wounds cytograms in the study group on the $10^{\text {th }}$ day of the post-operative period was 
proved as the regenerative-inflammatory in $24.81 \%$, regeneratory in $75.19 \%(p=0.031)$.

The chart of holiatry applied proved the efficiency of treatment of bad resistance cases of destructive polyonychomycosis by a subnail hyperkeratinization and secondary ingrowing toenail; the less traumatic removal of nails through onycholysis should be preferred, particularly after such treatment the patients with the type 2 diabetes mellitus experienced healing time of operative wound (crust formation) during 16-23 days and the indices tend to the control group.

\section{References}

1. Adamski Z, Kaszuba A. Dermatology for cosmetologists. Wroclaw: Elsevier Urban \& Partner; 2011;186.

2. Baran R, Feuithade M, Datry A. A randomized trial of amorolfine $5 \%$ nail lacquer combined with oral terbinafine compared with terbinafine alone in the treatment of dermatophytic toenail onychomycoses affecting the matrix region. Br J Dermatol. 200;142:1177-83.

3. Blaszczyk-Kostanecka M, Wolska H. Dermatology in practice. Warsaw: Medical Publishing House PZWL; 2009;230.

4. Brillowska-Dabrowska A, Saunte DM, Arendrup MC. Five hour diagnosis of dermatophytenail infections with specific detection of Trichophyton rubrum. J Clin Microbiol. 2007;45(4):1200-1204.

5. Brotzman SB, Wilk EC. Orthopedic Rehabilitation. Wroclaw: Elsevier Urban \& Partner; 2007;2(1):120.

6. Chang P. Onychogryphosis. Our Dermatol Online. 2011;2(4):227-28.

7. Chang P, Meaux T. Onychogryphosis: A Report of Ten Cases. Skinmed. 2015;13(5):355-59.

8. Chapeskie H, Kovac JR. Case Series: soft-tissue nail-fold excision: a definitive treatment for ingrown toenails. Can J Surg. 2010;53:282-86.

9. Demirseren DD, Emre S, Akoglu G, et al. Relationship between skin diseases and extracutaneous complications of diabetes mellitus: clinical analysis of 750 patients. Am J Clin Dermatol. 2014;15:65-70.

10. Gamboa A, Shibao C, Diedrich A, et al. Contribution of endothelial nitric oxide to blood pressure in humans. Hypertension. 2007;49(1):170-77.

11. Gupta AK, Baran R. Ciclopirox nail lacquer solution $8 \%$ in the 21 st century. J Am Acad Dermatol. 2000;43(4I):96-102.

12. Gupta AK, Gregurek-Novak T. Efficacy of itraconasole, terbinafine, fluconazole, griseofulvin, and ketoconazole in the treatment of Scopulariopsis brevicaulis causing onychomycosis of the toes. Dermatology. 2001;202:235-38.

13. Gupta AK, Schouten JR, Lynch LE. Ciclopirox Nail Lacquer 8\% for the Treatment of Onychomycosis: A Canadian Perspective. Skin Therapy Letter. 2005; 10(7):1-3.
14. Havu V, Heikkila $H$, Kuokkanen $K$, et al. A double-blind, randomized study to compare the efficacy and safety of terbinafine (Lamisil $\left.{ }^{\circledR}\right)$ with fluconazole (Diflucan $®$ ) in the treatment of onychomycosis. Br J Dermatol. 2000;142:97-102.

15. Khunger N, Kandhari R. Ingrown toenails. J Dermatol Venereol Leprol. 2012;78:279-89.

16. Lesher JL. Recent developments in antifungal therapy. Dermatol Clin. 2010;14(1):163-69.

17. Peyvandi $H$, Robati RM, Yegane RA, et al. Comparison of two surgical methods (winograd and sleeve method) in the treatment of ingrown toenail. Dermatol Surg. 2011;37:331-35.

18. Ragunatha S, Anitha B, Inamadar AC, et al. Cutaneous disorders in 500 diabetic patients attending diabetic clinic. Indian J ermatol. 2011;56:160-64.

19. Shemer A. Update: medical treatment of onychomycosis. Dermatologic Therapy. 2012;25: 582-93.

20. Tatlican S, Eren C, Yamangokturk B, et al. Chemical matricectomy with $10 \%$ sodium hydroxide for the treatment of ingrown toenails in people with diabetes. Dermatol Surg. 2010;36:219-22.

21. Thomas J, Jacobson GA, Narkowicz CK. Toenail onychomycosis: an important global disease burden. Journal of Clinical Pharmacy and Therapeutics. 2010;35:497-519.

22. Vergun AR. Multivitamin failure in patients with complicated destructive nail mycosis; analysis of clinical observations. 7 th Lviv-Lublin conference of Experimental and Clinical Biochemistry. Abstract book. Lviv. 2013. p. 176-77.

23. Welsh O. Vera-Cabrera L, Welsh E. Onychomycosis. Clinics in dermatology. 2010;28:151-59.

24. Yang KC, Li YT. Treatment of recurrent ingrown great toenail associated with granulation tissue by partial nail avulsion followed by matricectomy with sharpulse carbon dioxide laser. Dermatol Surg. 2002;28(5):419-21.

25. Yin Z, Xu J, Luo D. A metaanalysis comparing long term recurrences of toenail onychomycosis after successful treatment with terbinafine versus itraconazole. J Dermatol Treat. 2012;23(6):449-52. 